\title{
Fragmentation and mass segregation in the massive dense cores of Cygnus $X$
}

\author{
S. Bontemps ${ }^{1}$, F. Motte ${ }^{2}$, T. Csengeri ${ }^{2}$, and N. Schneider ${ }^{2}$ \\ ${ }^{1}$ Université de Bordeaux, Laboratoire d'Astrophysique de Bordeaux, CNRS/INSU, UMR 5804, BP 89, 33271 Floirac Cedex, France \\ e-mail: bontemps@obs.u-bordeaux1.fr \\ 2 Laboratoire AIM, CEA/DSM, IRFU/Service d'Astrophysique, 91191 Gif-sur-Yvette Cedex, France
}

Received 11 September 2009 / Accepted 21 July 2010

\begin{abstract}
Massive dense cores (MDCs) are the high-mass equivalent of the so-called dense cores in nearby star-forming regions. With typical sizes of $0.1 \mathrm{pc}$, they could form either a few high-mass stars, or a cluster of low-mass stars. We present high-angular resolution continuum observations obtained with the IRAM Plateau de Bure interferometer at 1.3 and $3.5 \mathrm{~mm}$ towards the six most massive and youngest (IR-quiet) dense cores in the Cygnus X complex. Located at only $1.7 \mathrm{kpc}$, the Cygnus X region offers the opportunity of reaching small enough scales (of the order of $1700 \mathrm{AU}$ at $1.3 \mathrm{~mm}$ ) to separate individual collapsing objects, and thus to observe and constrain the result of the fragmentation process. The cores are sub-fragmented with a total of 23 fragments inside 5 cores. Only the most compact MDC, CygX-N63, may host a single proto-stellar object with an envelope as massive as $\sim 60 M_{\odot}$. The fragments in the other cores have sizes and separations similar to low-mass pre-stellar condensations and Class 0 young stellar objects in nearby protoclusters, and are most probably self-gravitating objects $\left(M>M_{\text {vir }}\right)$. In addition to CygX-N63, a total of 8 objects are found to be probable precursors of $\mathrm{OB}$ stars with their envelope masses ranging from 8.4 to $30 M_{\odot}$ inside a $F W H M$ of 4000 AU. The level of fragmentation is globally higher than in the turbulence regulated, monolithic collapse scenario, but it is also not as high as expected in a pure gravo-turbulent scenario where the distribution of mass is dominated by low-mass protostars/stars. Here, the fractions of the total MDC masses in the high-mass proto-stellar fragments are found to be as high as 37, 58, and 100\% in CygX-N12, CygX-N53, and CygX-N63, respectively. These high fractions of mass in the proto-stellar fragments are also indicative of a high efficiency of core formation in the MDCs. The increase in the core formation efficiency as a function of average density in the MDCs is proposed to be caused by the increasing importance of self-gravity leading to gravitational collapse on the scale of the MDCs. At the same time, the observed MDCs tend to fragment into a few proto-stellar objects within their central regions. We are therefore probably witnessing the primordial mass segregation of clusters. The physical origin of the fragmentation into a few high-mass objects is not yet clear, and will be investigated in the future by studying the kinematics of the MDCs.
\end{abstract}

Key words. stars: formation - stars: luminosity function, mass function - ISM: clouds

\section{Introduction}

The detailed physical processes at the origin of high-mass stars (here defined as ionizing OB stars; i.e., earlier than B3 or $M_{\star}>$ $8 M_{\odot}$ ) are still to be fully recognized and understood. Whether they form through a scaled-up version of the low-mass star formation process or by means of a specific process is still an open issue. The formation process might be different for two main reasons. First, the radiation pressure of the ionizing protostar may stop accretion when it reaches a mass of $\sim 10 M_{\odot}$. With a moderate accretion rate $\left(\sim 10^{-5} M_{\odot} / \mathrm{yr}\right)$, the star could reach at most $\sim 40 M_{\odot}$ (see Yorke \& Sonnhalter 2002, and references therein). Second, OB stars are found to form in clusters and may be formed by collective effects. Competitive accretion is one of the scenarios that require cluster forming conditions because the accretion efficiencies and rates depend on the gravitational potential of the protocluster (Bonnell \& Bate 2006). The radiation pressure may not be a problem if the accretion rates are in practice much higher than usually measured in low-mass protostars (typically between $10^{-6}$ and $10^{-5} M_{\odot} / y r$ ). High infall rates could be expected in the classical theory of star formation applied to low-mass stars if the initial conditions for collapse depart significantly from a singular $r^{-2}$ sphere or if the collapsing conditions are reached in a dynamical way (see Larson 1969; Whitworth \& Summers 1985; Foster \& Chevalier 1993; Henriksen et al. 1997; Chabrier 2003). Alternatively, McKee \& Tan (2002) propose that even in a slow, quasi-static evolution of star-forming clumps, high accretion rates could be due to the high level of turbulent support. A continuous injection of turbulence in the high density regions is then required. Turbulence is also usually believed to be at the origin of the hierarchical structure of molecular clouds (e.g., Larson 1981; Falgarone et al. 2004) and may therefore regulate the fragmentation of clouds from large scales down to collapsing cores (see Vazquez-Semadeni 1994; Padoan \& Nordlund 2002; Chabrier 2003). The turbulent fragmentation can adequately reproduce the observed core mass functions, which mimic the initial mass function (IMF) of stars (Klessen 2001; Hennebelle \& Chabrier 2008). In this description, the most massive stars would originate from the rare highmass fragments that could decouple from their turbulent environment. In a purely gravitational view, the typical stellar masses are dictated by the Jeans masses that are usually of the order of a fraction of a solar mass. This can explain the average stellar masses, but has problems in explaining the formation of stars of the highest masses. The latter are often two orders of magnitude higher than the Jeans masses. Some additional effects are needed 
to fully understand how fragmentation proceeds to produce massive cores, or to enable specific conditions to grow massive stars from low-mass collapsing fragments.

The final fragmentation leading to individual collapsing protostars inside the dense massive clumps is therefore one of the central unknowns in our understanding of the origin of massive stars. In the slow evolution of turbulent clouds promoted by McKee \& Tan (2002), the level of fragmentation is lower and should lead to stable (relatively long-living) massive cores that would collapse into a single star or a multiple bound system. In the dynamical view, the clumps are expected to fragment into a large number of low-mass fragments (e.g. Bonnell et al. 2007), which may grow in mass in the center of the forming cluster when the gravitational well is dominated by the protostellar and stellar components. The fragmentation inside the most massive dense cores on the scale of a few $0.1 \mathrm{pc}$, i.e. the most likely candidates to be protoclusters in their earliest phases, can help us to discriminate between the theoretical schemes in competition.

Since massive stars are rare, and the lifetime of their earliest phases is short, the protostellar precursors of massive stars remain elusive objects. Large-scale systematic surveys are mandatory to find them. The early works obtained in the vicinity of luminous IRAS or maser sources in the whole Galactic plane (Molinari et al. 1996; Plume et al. 1997; Beuther et al. 2002; Mueller et al. 2002; Faúndez et al. 2004), and inside the dark filaments seen in the infrared surveys of the Galactic plane, the so-called IRDCs (e.g. Hennebelle et al. 2001; Simon et al. 2006; Peretto \& Fuller 2009) found a number of candidates to host these precursors (e.g. Rathborne et al. 2006; Pillai et al. 2006). These objects are however spread over the whole Galaxy, at distances usually larger than $3 \mathrm{kpc}$, and their detection is not based on complete imaging of star-forming complexes, which preclude us obtaining a representative view of these poorly studied cold phases. In Cygnus X, Motte et al. (2007) did perform a complete survey of a molecular complex rich enough to provide a sample of massive dense cores (hereafter MDCs). Cygnus X is a nearby massive complex at a distance of only $1.7 \mathrm{kpc}$ (Schneider et al. 2006, 2007). A total of 40 MDCs have been discovered. These cores are dense, and massive on a scale of $\sim 0.1 \mathrm{pc}$ and can thus be expected to be representative of the earliest phases of the formation of high-mass stars. Even the MDCs, which are not seen as dark silhouettes on the IR background (IRDCs), are recognized. Following the nomenclature of Williams et al. (2000), we note that, in terms of sizes, the $\sim 0.1$ pc MDCs of Cygnus $\mathrm{X}$ should be considered as dense cores but may not collapse into a single star. We here adopt the classical term of dense cores for the $\sim 0.1 \mathrm{pc}$ entities even if they might be sub-fragmented and may form a small cluster of stars. The terms fragments or condensations is then used for smaller structures on the scale of a few $0.01 \mathrm{pc}$ inside these dense cores, which may correspond to individual pre-stellar or proto-stellar condensations such as those found in the nearby and low-mass clusters in formation (e.g. Motte et al. 1998),

We present the first results of a systematic study at high spatial resolution of the MDCs of Cygnus X. The 1 and $3 \mathrm{~mm}$ continuum maps of 6 IR-quiet MDCs obtained with the Plateau de Bure interferometer are discussed. The accompanying line emission obtained at the same time will be included in forthcoming papers dedicated to outflows for the $\mathrm{CO}$ and $\mathrm{SiO}$ lines, and to the dense molecular gas inside the MDCs for the $\mathrm{H}^{13} \mathrm{CO}^{+}$ and $\mathrm{H}^{13} \mathrm{CN}$ lines (Csengeri et al. 2010). The source selection and the observations are explained in Sect. 2. The continuum maps as well as pointed observations in $\mathrm{N}_{2} \mathrm{H}^{+}$towards each MDCs obtained with the IRAM $30 \mathrm{~m}$ telescope are shown in Sect. 3.
In Sect. 4, we discuss the inferred fragmentation properties of these MDCs in the framework of understanding the origin of high-mass stars.

\section{Source selection and observations}

\subsection{IR-quiet MDCs in Cygnus $X$}

From the complete imaging survey of the high column density regions of Cygnus $\mathrm{X}$ in the $1.3 \mathrm{~mm}$ continuum with MAMBO on the IRAM $30 \mathrm{~m}$ telescope, Motte et al. (2007) recognized 129 dense cores with masses ranging from 4 to $950 M_{\odot}$. Among these, a total of 40 were found to be more massive than $40 M_{\odot}$ and identified as probable precursors of high-mass stars in Cygnus X. With an average size of $0.13 \mathrm{pc}$ and an average density of $1.9 \times 10^{5} \mathrm{~cm}^{-3}$, these MDCs have sizes similar to those of low-mass dense cores in nearby molecular clouds (Ward-Thompson et al. 1994; Bacmann et al. 2000; Evans et al. 2001; Ward-Thompson et al. 2007). However they are on average almost 10 times denser and 20 times more massive than their low-mass counterparts (see Table 4 in Motte et al. 2007). Despite their high densities and masses, which should lead to intense star formation activity, as many as 17 out of 40 cores do not emit or emit only weakly in the mid-infrared (IR-quiet). This lack of strong infrared emission may indicate that these regions are not yet forming massive stars. On the other hand, strong SiO emission is systematically detected towards them. The intensities and profiles of these $\mathrm{SiO}$ lines are indicative of powerful outflows most probably driven by high-mass protostars. These properties are very similar to the properties of low-mass Class 0 young stellar objects (hereafter YSOs), which are dense, cold objects driving powerful outflows (e.g. André et al. 1993). A critical comparison suggests that the IR-quiet MDCs are either a scaled-up version (in size and density) of low to intermediate mass Class 0 YSOs or correspond to clusters with a significant number of lowmass Class 0 YSOs.

\subsection{A representative sample of 6 IR-quiet MDCs}

We selected the 6 most massive IR-quiet MDCs of Cygnus X. The names and coordinates from Motte et al. (2007) are listed in Table 1. An overview of the locations of these MDCs is given in Fig. 1. Three of the cores (CygX-N40, CygX-N48, and CygX-N53) are located in the DR21 filament, which is the most massive and dense part of Cygnus $\mathrm{X}$, and a wellknown region of massive star formation (Schneider et al. 2010). CygX-N40 and CygX-N48 correspond to the submillimeter sources DR21(OH)-N2 and DR21(OH)-S, respectively, while CygX-N53 is situated close to FIR3 (Chandler et al. 1993). The other 3 selected MDCs were discovered by the MAMBO survey and are situated in more isolated, but still prominent molecular clumps bright in CS emission (Fig. 1). In the western part, CygX-N3 is located close to DR17, which is an HII region excited by two OB clusters (clusters \# 12 and 14 in Le Duigou \& Knödlseder 2002). These clusters shape the cloud and CygX-N3 corresponds to the tip of a pillar-like cloud. CygX-N12 is also situated in a cometary shape cloud probably influenced by the same OB clusters but the cloud seems to be less compressed from outside than CygX-N3. Finally, CygX-N63 appears to be located in the south of DR21 in the DR22-DR23 filament (see Schneider et al. 2006). It can be considered as the most isolated MDC of the sample. 
Table 1. Observing parameters and obtained beam sizes and rms.

\begin{tabular}{ccccccc}
\hline \hline & \multicolumn{2}{c}{$\begin{array}{c}\text { Phase center } \\
\text { Source }\end{array}$} & \multicolumn{2}{c}{$\begin{array}{c}\text { Synthesized beam } \\
{[\operatorname{arcsec} \times \operatorname{arcsec}]}\end{array}$} & \multicolumn{2}{c}{$\begin{array}{c}\text { Obtained rms } \\
{[\mathrm{mJy} / \mathrm{beam}]}\end{array}$} \\
name & RA & Dec & $1.3 \mathrm{~mm}$ & $3.5 \mathrm{~mm}$ & $1.3 \mathrm{~mm}$ & $3.5 \mathrm{~mm}$ \\
\hline CygX-N3 & 203534.1 & 422005.0 & $1.09 \times 0.92$ & $2.66 \times 2.20$ & 1.2 & 0.21 \\
CygX-N12 & 203657.4 & 421127.5 & $1.14 \times 0.83$ & $3.02 \times 2.28$ & 1.9 & 0.22 \\
CygX-N40 & 203859.8 & 422342.0 & $1.08 \times 0.92$ & $2.62 \times 2.17$ & 1.0 & 0.17 \\
CygX-N48 & 203901.5 & 422204.0 & $1.12 \times 0.91$ & $2.81 \times 2.33$ & 2.2 & 0.34 \\
CygX-N53 & 203903.1 & 422550.0 & $1.05 \times 0.87$ & $3.16 \times 2.57$ & 1.9 & 0.25 \\
CygX-N63 & 204005.2 & 413212.0 & $1.15 \times 0.83$ & $3.05 \times 2.24$ & 4.2 & 0.33 \\
\hline
\end{tabular}

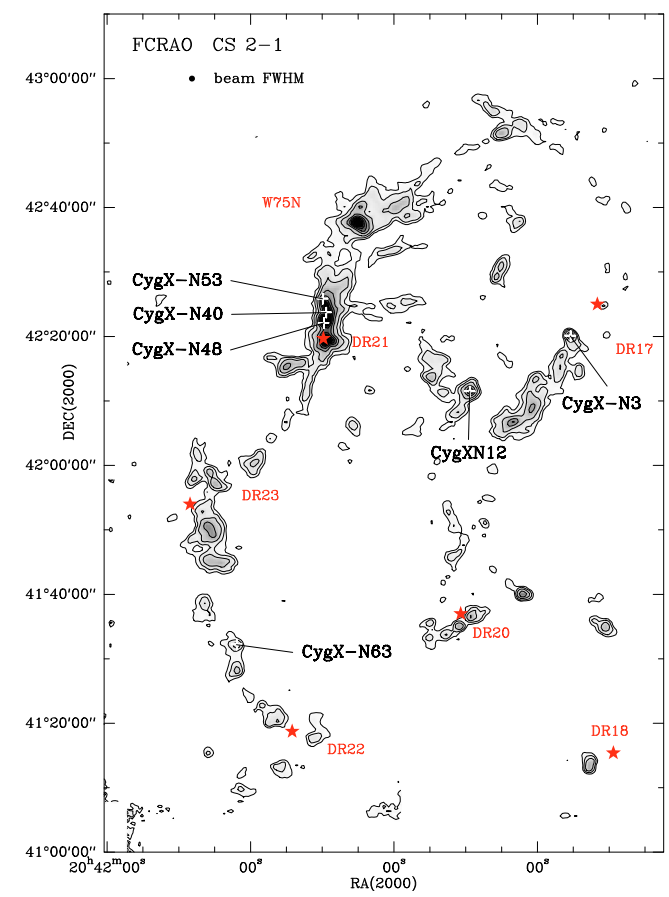

Fig. 1. Overview of the northern part of Cygnus $\mathrm{X}$ indicating the location of the 6 MDCs from Motte et al. (2007). The contour map displays the integrated emission in $\mathrm{CS}(2-1)$ as observed with the FCRAO (Schneider et al. 2010). Note that 3 out of the 6 MDCs (CygX-N40, CygX-N48, and CygX-N53) are clustered within the most massive region of Cygnus X, the DR21 filament. The red stars indicate the centers of the HII regions DR17 to DR23 from Downes \& Rinehart (1966).

\subsection{Interferometric observations of the 1 and $3 \mathrm{~mm}$ continuum}

We used the IRAM ${ }^{1}$ Plateau de Bure Interferometer (hereafter PdBI) to image the dust continuum at high resolution simultaneously at 3.5 and $1.3 \mathrm{~mm}$. The $1 \mathrm{~mm}$ and $3 \mathrm{~mm}$ receivers were tuned to 230.54 and $86.61 \mathrm{GHz}$, respectively, with two $320 \mathrm{MHz}$ units placed at 230.41 and $230.67 \mathrm{GHz}$ and 86.48 and $86.74 \mathrm{GHz}$, respectively. In addition to the continuum, the emissions in 4 molecular lines were also observed, i.e., ${ }^{12} \mathrm{CO}(2-1)$, $\mathrm{SiO}(2-1), \mathrm{H}^{13} \mathrm{CO}^{+}(1-0)$, and $\mathrm{H}^{13} \mathrm{CN}(1-0)$ at $230.54,86.85$, 86.75 , and $86.34 \mathrm{GHz}$, respectively. These line data will be presented in forthcoming papers.

The observations were performed in track-sharing mode with two targets per track for the following pairs: CygX-N48/ CygX-N53, CygX-N3/CygX-N40, and CygX-N12/CygX-N63. The $\mathrm{D}$ configuration track observations were performed between

\footnotetext{
${ }^{1}$ IRAM is supported by INSU/CNRS (France), MPG (Germany), and IGN (Spain).
}

June and October 2004 with 5 antennas with baselines ranging from 24 to $82 \mathrm{~m}$. The $\mathrm{C}$ configuration tracks were obtained in November and December 2004 with 6 antennas in 6Cp with baselines ranging from 48 to $229 \mathrm{~m}$. As a phase calibrator, we mostly used the bright nearby quasar $2013+370$ and as a flux calibrator the evolved star MWC 349, which is located in Cygnus X.

To enhance the spatial resolution, the maps were cleaned using the uniform weighting. The resulting synthesized beam and rms in the continuum are summarized in Table 1 together with the field names and centers of phase. The cleaning components were searched across the whole area of the primary beams. No polygon was used to avoid introducing any bias in the resulting maps of emission.

\subsection{IRAM 30 m observations of $\mathrm{N}_{2} \mathrm{H}^{+} 1 \rightarrow 0$}

The $\mathrm{N}_{2} \mathrm{H}^{+} \quad 1 \rightarrow 0$ line at $93.176265 \mathrm{GHz}$ was observed in September 2003 using the B100 receiver and the VESPA correlator at the IRAM $30 \mathrm{~m}$ telescope. The average system temperature was $182 \mathrm{~K}$ and the average rms of the spectra is $0.19 \mathrm{~K}$. The data are corrected for the main beam efficiency of 0.78 and have a velocity resolution of $0.13 \mathrm{~km} \mathrm{~s}^{-1}$, and a beamsize of $29^{\prime \prime}$.

\section{Results}

\subsection{The $3 \mathrm{~mm}$ continuum maps}

The left panels in Figs. 2 and 3 display the dust continuum emission towards the six cores at $3.5 \mathrm{~mm}$. Strong $3 \mathrm{~mm}$ emission is detected for all fields except CygX-N40. Despite a total mass of $106 M_{\odot}$ in Motte et al. (2007), CygX-N40 seems to be dominated by extended emission, which is fully filtered out by the interferometer. We note that it has the largest size and consequently the lowest average density in our sample, which implies that this core is not yet concentrated if it is much younger than the other cores. Alternatively, since CygX-N40 is located in the large-scale DR21 filament, the observed emission may be more elongated along the line-of-sight, leading to an overestimation of the local density and to a misinterpretation of CygX-N40 as a single MDC. In any case, it seems that this core is of dissimilar nature to the rest of the sample, and it will therefore not be further considered for discussion. For the other fields, the emission contains bright compact emission peaks. They are structured into a few individual bright compact sources - except CygX-N63, which seems to be dominated by a single object. We also note that $\mathrm{CygX}-\mathrm{N} 48$ appears to be less centrally concentrated than the other cores, and may be dominated by a large population of unresolved compact fragments. 

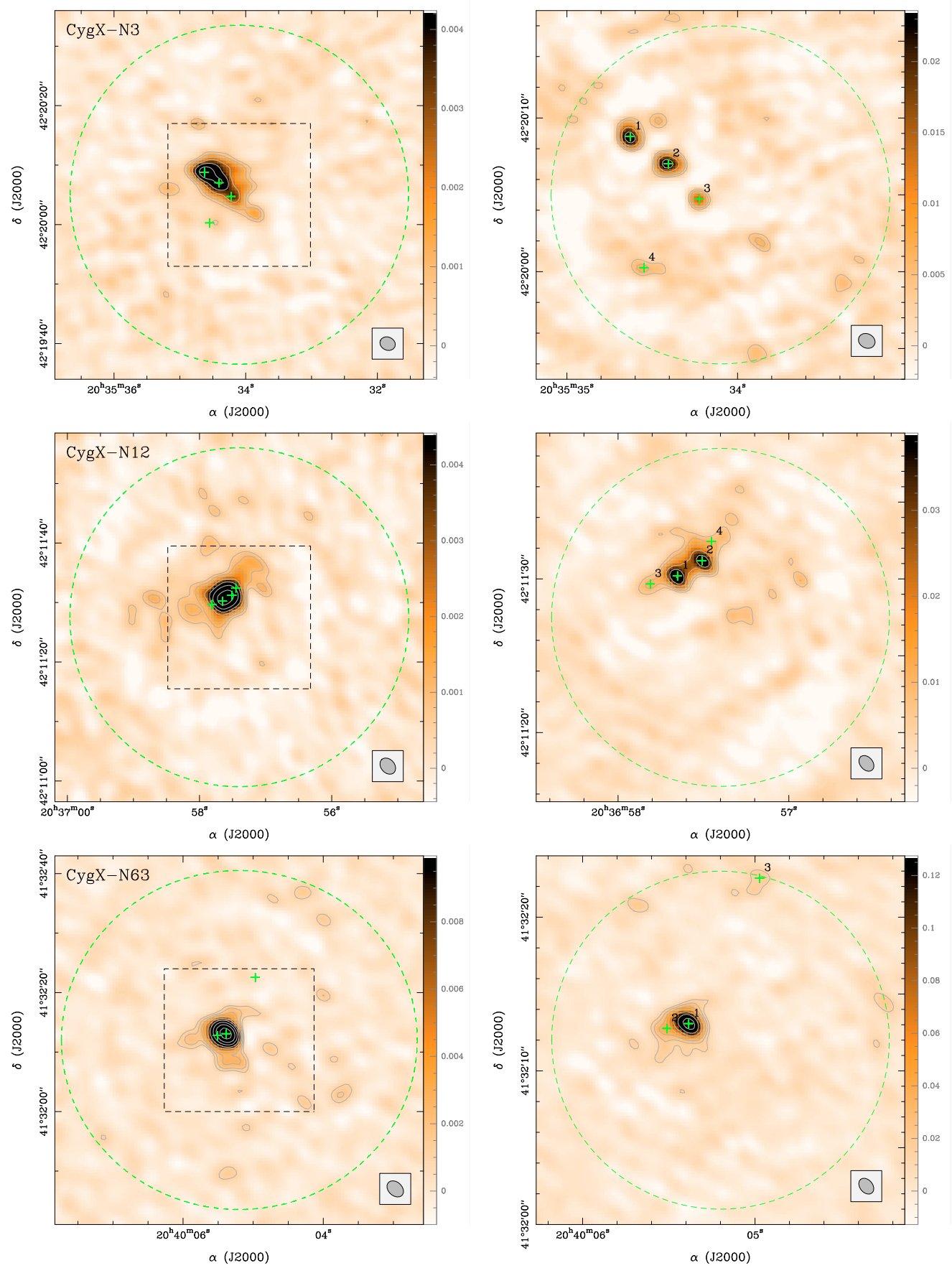

Fig. 2. Maps of the continuum at $3.5 \mathrm{~mm}$ (left) and $1.3 \mathrm{~mm}$ (right) obtained with the PdBI for the three more isolated MDCs (see text). The color images have linear scales from $-2 \sigma$ to $+20 \sigma$ for the 4 top panels, and from $-3 \sigma$ to $+30 \sigma$ for the 2 lower ones where $\sigma$ is the resulting rms in each field (see Table 1) (the unit on the right of the plots is Jy/beam). The (logarithmic) contour levels are $3 \sigma, 4.8 \sigma, 7.5 \sigma, 12 \sigma, 19 \sigma, 30 \sigma, 75 \sigma$, and $120 \sigma$. The crosses and numbers indicate the locations and the names of the fragments at $1 \mathrm{~mm}$ (see Table 2). The synthetised beams are displayed in the bottom right corners. The dashed circles indicate the primary beams. In the $3.5 \mathrm{~mm}$ maps (left), the name of the MDC is given, and the dashed squares indicate the areas covered at $1.3 \mathrm{~mm}($ right). Note the similar behavior of the 3 MDCs. A compact core is observed at $3.5 \mathrm{~mm}$ that separates into smaller fragments at $1.3 \mathrm{~mm}$. CygX-N63 seems to actually correspond to a single object down to the $1^{\prime \prime}$ (1700 AU) spatial resolution.

\subsection{The $1 \mathrm{~mm}$ continuum maps}

The right panels in Figs. 2 and 3 display the dust continuum maps at $1 \mathrm{~mm}$. For three of the cores (CygX-N3, CygX-N12, CygX-N53), the difference between low $(3.5 \mathrm{~mm})$ and high $(1.3 \mathrm{~mm})$ resolution maps is striking. While on a scale of $2^{\prime \prime}$ to $3^{\prime \prime}$ resolution, the emission consists mainly of extended, elongated structures, at the $\sim 1^{\prime \prime}$ resolution of the $1.3 \mathrm{~mm}$ map the emission clearly separates into a number of individual usually spherical fragments. In contrast, $\mathrm{CygX}-\mathrm{N} 48$ has a similar aspect at $1.3 \mathrm{~mm}$ as at $3.5 \mathrm{~mm}$ with emission being spread over the whole region and compact sources not being very spherical implying that it consists of partly non-resolved individual objects. CygX-N63 seems to also differ from the others since the emission is dominated on all observed scales by a single central object. In the other MDCs, some distributions of aligned 
S. Bontemps et al.: Fragmentation in the massive dense cores of Cygnus $\mathrm{X}$
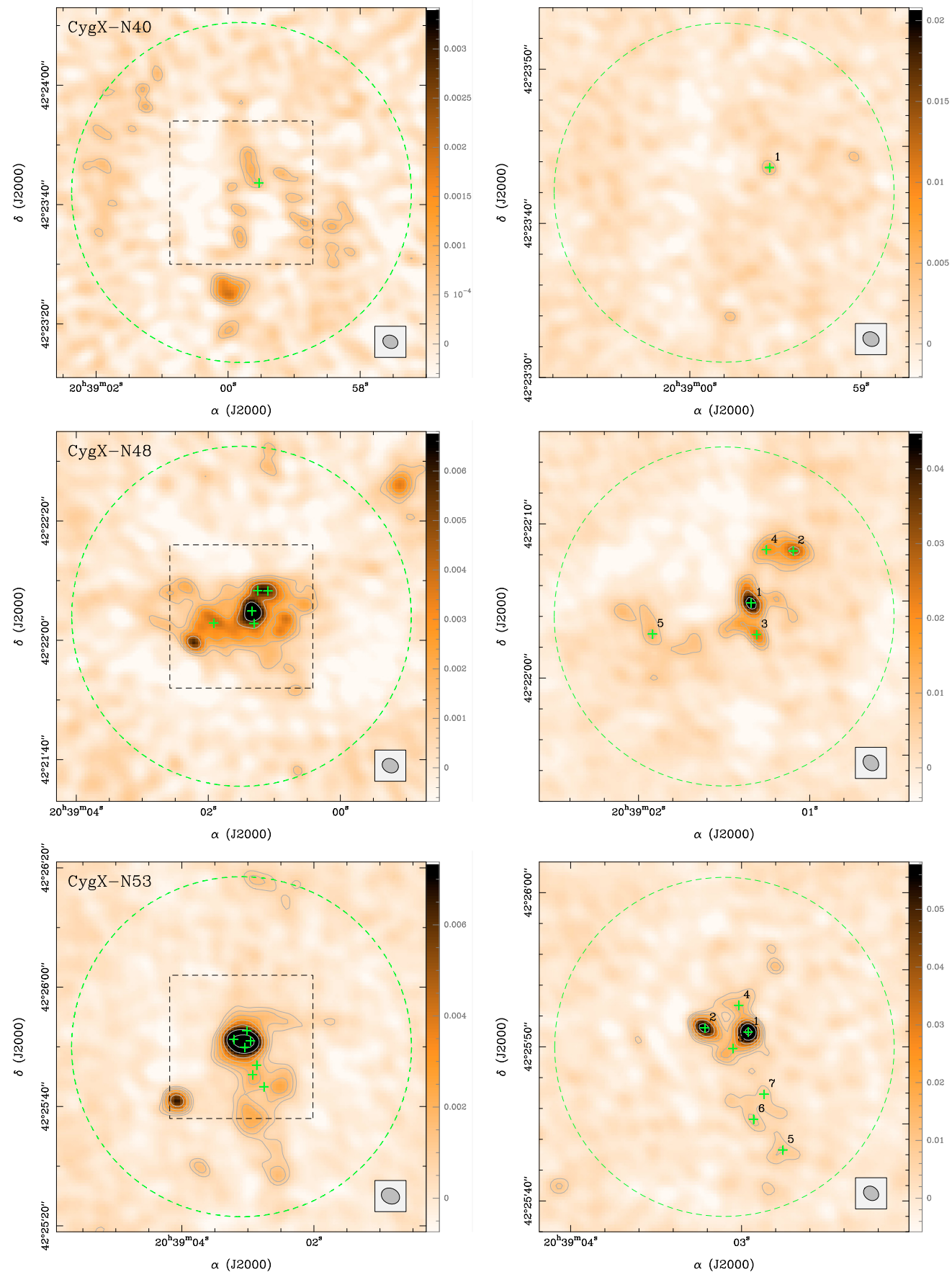

Fig. 3. Same as Fig. 2 for the three MDCs located in the filament of DR21 (see text). Note that in contrast to the isolated MDCs (Fig. 2), the cores show here diverse structures. CygX-N40 appears devoid of compact emission at both 3.5 and $1.3 \mathrm{~mm}$. CygX-N48 has a complex and extended structure at $3.5 \mathrm{~mm}$ with two main cores in the central regions. The $1.3 \mathrm{~mm}$ emission resolves a few, probable compact, fragments in the two main cores. CygX-N53 has a bright central core at $3.5 \mathrm{~mm}$ that divides into 4 smaller fragments at $1.3 \mathrm{~mm}$ in a way similar to the isolated MDCs. The bright peak at $3.5 \mathrm{~mm}$ in the south-east corresponds to the weak $1.3 \mathrm{~mm}$ source outside in the primary beam (dashed circle) in the right panel. It is located at the edge of the nearby cluster associated with MSX6G81.7133+0.5589.

compact sources inside filamentary structures appear to be seen at $3.5 \mathrm{~mm}$. CygX-N3 and CygX-N12 are composed of three to four aligned compact sources. CygX-N48 has an "S" shape emission, which may also be a filamentary structure. CygX-N53 displays an almost north-south ridge of emission that is aligned with the large-scale orientation of the DR21 filament in which it is embedded. The direction of the axis between the two brightest sources is however oriented east-west, almost perpendicular to the main direction of the large-scale emission.

\subsection{Source extraction at $1 \mathrm{~mm}$}

Our ultimate goal is to study the population of individual protostellar objects inside the MDCs of Cygnus X. We therefore extract fragments from the highest spatial resolution data maps ( $1 \mathrm{~mm}$ maps). To be systematic, we used the Gaussclump algorithm (Stutzki \& Guesten 1990) adapted to continuum maps as described in Motte et al. (2007) to extract the compact sources. Above a safe $7 \sigma$ level, 24 sources have been detected in the 
Table 2. List of fragments and physical properties at $1.3 \mathrm{~mm}$.

\begin{tabular}{|c|c|c|c|c|c|c|c|c|c|}
\hline \multirow{2}{*}{$\begin{array}{l}\text { Fragment } \\
\text { name }\end{array}$} & \multicolumn{2}{|c|}{ Coordinates } & \multirow{2}{*}{$\begin{array}{c}\text { Peak } \\
\text { flux } \\
{[\mathrm{mJy} / \mathrm{beam}]}\end{array}$} & \multirow{2}{*}{$\begin{array}{c}F W H M^{a} \\
{\left[{ }^{\prime \prime} \times{ }^{\prime \prime}\right]}\end{array}$} & \multirow{2}{*}{$\begin{array}{c}\text { Int. } \\
\text { flux } \\
{[\mathrm{mJy}]}\end{array}$} & \multirow{2}{*}{$\begin{array}{c}M_{\mathrm{PdBI}}^{b} \\
{\left[M_{\odot}\right]} \\
\end{array}$} & \multirow{2}{*}{$\begin{array}{c}\text { Deconv. }^{c} \\
\text { size } \\
{[\mathrm{AU}]}\end{array}$} & \multirow{2}{*}{$\begin{array}{l}\left\langle n_{\mathrm{H}_{2}}\right\rangle^{d} \\
{\left[\mathrm{~cm}^{-3}\right]}\end{array}$} & \multirow{2}{*}{$\begin{array}{l}M_{\mathrm{env}}^{e} \\
{\left[M_{\odot}\right]}\end{array}$} \\
\hline & $\begin{array}{c}\text { RA } \\
(\mathrm{J} 2000) \\
\end{array}$ & $\begin{array}{c}\text { Dec } \\
(\mathrm{J} 2000) \\
\end{array}$ & & & & & & & \\
\hline CygX-N3 MM1 & 203534.63 & 422008.8 & 37.3 & $1.27 \times 1.05$ & 49.6 & 2.63 & 979 & $1.48 \times 10^{8}$ & $8.1-13.4$ \\
\hline CygX-N3 MM2 & 203534.41 & 422007.0 & 30.2 & $1.34 \times 1.04$ & 41.6 & 2.21 & 1050 & $1.01 \times 10^{8}$ & $6.3-10.5$ \\
\hline CygX-N3 MM3 & 203534.23 & 422004.7 & 14.4 & $1.01 \times 1.00$ & 14.5 & 0.77 & $<851$ & $>6.56 \times 10^{7}$ & $2.7-4.5$ \\
\hline CygX-N3 MM4 & 203534.55 & 422000.3 & 8.7 & $2.01 \times 1.01$ & 17.7 & 0.94 & 1720 & $9.65 \times 10^{6}$ & $1.6-2.7$ \\
\hline CygX-N12 MM1 & 203657.65 & 421130.2 & 64.1 & $1.22 \times 1.12$ & 92.6 & 4.91 & 1100 & $1.93 \times 10^{8}$ & $13.4-22.3$ \\
\hline CygX-N12 MM2 & 203657.51 & 421131.2 & 54.7 & $1.46 \times 1.13$ & 95.2 & 5.05 & 1420 & $9.23 \times 10^{7}$ & $10.7-17.8$ \\
\hline CygX-N12 MM3 & 203657.81 & 421129.7 & 12.4 & $1.77 \times 1.19$ & 27.4 & 1.45 & 1820 & $1.26 \times 10^{7}$ & $2.4-4.0$ \\
\hline CygX-N12 MM4 & 203657.45 & 421132.4 & 11.7 & $3.11 \times 0.98$ & 37.6 & 1.99 & 2460 & $7.00 \times 10^{6}$ & $2.4-4.0$ \\
\hline CygX-N40 MM1 & 203859.54 & 422343.6 & 5.7 & $1.04 \times 1.00$ & 5.9 & 0.31 & $<847$ & $>2.72 \times 10^{7}$ & $1.1-1.9$ \\
\hline CygX-N48 MM1 & 203901.34 & 422204.9 & 53.2 & & 88.5 & & 1400 & & $10.1-16.8$ \\
\hline CygX-N48 MM2 & 203901.10 & 422208.3 & 37.5 & $1.84 \times 1.28$ & 86.6 & 4.59 & 1960 & $3.19 \times 10^{7}$ & $7.0-11.7$ \\
\hline CygX-N48 MM3 & 203901.31 & 422202.8 & 25.6 & $1.65 \times 1.01$ & 41.7 & 2.21 & 1360 & $4.63 \times 10^{7}$ & $4.9-8.1$ \\
\hline CygX-N48 MM4 & 203901.25 & 422208.3 & 20.0 & $1.64 \times 1.14$ & & & 1570 & $2.65 \times 10^{7}$ & $3.7-6.2$ \\
\hline CygX-N48 MM5 & 203901.92 & 422202.9 & 12.8 & $2.34 \times 1.24$ & 36.3 & 1.93 & 2330 & $8.04 \times 10^{6}$ & $2.5-4.1$ \\
\hline CygX-N53 MM1 & 203902.96 & 422551.0 & 106.3 & $1.19 \times 1.09$ & & 8.00 & 1050 & $3.61 \times 10^{8}$ & $22.8-38.0$ \\
\hline CygX-N53 MM2 & 203903.22 & 422551.2 & 71.1 & $1.39 \times 1.03$ & 112.1 & 5.94 & 1230 & $1.66 \times 10^{8}$ & $14.4-24.1$ \\
\hline CygX-N53 MM3 & 203903.05 & 422549.9 & 17.3 & $1.46 \times 1.45$ & 40.2 & 2.13 & 1870 & $1.72 \times 10^{7}$ & $3.4-5.7$ \\
\hline CygX-N53 MM4 & 203903.02 & 422552.7 & 16.2 & $1.44 \times 1.44$ & 37.0 & 1.96 & 1840 & $1.66 \times 10^{7}$ & $3.2-5.3$ \\
\hline CygX-N53 MM5 & 203902.76 & 422543.3 & 13.2 & $1.72 \times 1.60$ & 39.8 & 2.11 & 2300 & $9.08 \times 10^{6}$ & $2.7-4.6$ \\
\hline CygX-N53 MM6 & 203902.93 & 422545.3 & 11.8 & $2.21 \times 1.02$ & 29.4 & 1.56 & 1980 & $1.06 \times 10^{7}$ & $2.4-3.9$ \\
\hline CygX-N53 MM7 & 203902.87 & 422546.9 & 9.5 & $2.27 \times 1.07$ & 25.3 & 1.34 & 2100 & $7.60 \times 10^{6}$ & $1.9-3.2$ \\
\hline CygX-N63 MM1 & 204005.39 & 413213.1 & 274.3 & & 419.2 & 22.22 & 1210 & $6.64 \times 10^{8}$ & $55.2-92.0$ \\
\hline CygX-N63 MM2 & 204005.51 & 413212.7 & 44.1 & $2.09 \times 1.44$ & 139.4 & 7.39 & 2440 & $2.68 \times 10^{7}$ & $9.1-15.2$ \\
\hline CygX-N63 MM3 & 204004.97 & 413222.5 & 37.8 & $1.63 \times 1.49$ & 96.1 & 5.09 & 2060 & $3.05 \times 10^{7}$ & $7.4-12.4$ \\
\hline
\end{tabular}

Notes. ${ }^{(a)}$ Not deconvolved $F W H M$ of the 2 D Gaussian. ${ }^{(b)}$ Mass derived from the PdBI integrated flux. ${ }^{(c)}$ Deconvolved $F W H M$ in $1 D(q u a d r a t i c$ average of the 2 axis sizes) expressed in AU for $d=1.7 \mathrm{kpc} .^{(d)}$ The average density is calculated inside the projected region containing $90 \%$ of the mass in a 2D Gaussian assuming a spherical distribution. ${ }^{(e)}$ Range of $M_{\mathrm{env}}$ extrapolated from $M_{\mathrm{PdBI}}$ to $F W H M$ sizes of 3000 to $5000 \mathrm{AU}$ assuming a density profile in $r^{-2}$ (see Sect. 3.3 for details).

6 fields and 23 in 5 fields (excluding CygX-N40), i.e. an average of almost 5 sources per field. All sources were able to be clearly identified by eye-inspection in the maps.

The obtained list of fragments, ordered by increasing peak flux, is given in Table 2. The table gives the coordinates, peak fluxes, 2D Gaussian sizes (FWHM), and integrated fluxes directly obtained from the Gausclump analysis. The fraction of total (single-dish) flux recovered by the PdBI can be evaluated by comparing the integrated fluxes by Motte et al. (2007) with the integrated emission above $3 \sigma$ associated with all sources detected at a $5 \sigma$ level. The obtained values are 9 and $13 \%$ for CygX-N3 and CygX-N48, respectively, which are rather typical of these interferometric observations. However, it reaches higher, and more exceptional values of 20,34 , and $61 \%$ for the three most compact cores, CygX-N12, CygX-N53, and CygX-N63, respectively. The integrated fluxes are converted into masses $M_{\mathrm{PdBI}}$ by assuming that the dust emission is optically thin and adopting a dust emissivity at $1.3 \mathrm{~mm}$ of $1.0 \mathrm{~cm}^{2} \mathrm{~g}^{-1}$ (Ossenkopf \& Henning 1994), a gas-to-dust mass ratio of 100, and a dust temperature of $20 \mathrm{~K}$ (formula (1) in Motte et al. 2007). The deconvolved sizes (Col. 8 in Table 2) correspond to the quadratic average of the deconvolved 2D sizes ( FWHM) and are converted into AU for $d=1.7 \mathrm{kpc}$. Average densities are calculated inside the projected region of radius $R_{90}$ containing $90 \%$ of the mass $\left(M_{\mathrm{PdBI}}\right)$ for a $2 \mathrm{D}$ Gaussian distribution $\left(R_{90}=1.95 \times F W H M / \sqrt{8 \ln 2}\right)$. The masses $M_{\mathrm{PdBI}}$ obtained from the Gaussian decomposition of the PdBI maps are good estimates of the masses inside the inner regions of the compact fragments. Their true sizes and total masses cannot however be easily derived from the present interferometric data, which tend to filter out the extended envelopes (see Sect. 4.3). An estimate of the total envelope masses $M_{\text {env }}$ is given in Table 2. This is an extrapolation of $M_{\mathrm{PdBI}}$ inside a size $(F W H M)$ of $4000 \pm$ 1000 AU. According to the detailed discussion given in Sect. 4.3, these masses should be seen as conservative estimates of the total masses of the protostellar envelopes in contrast to the values of $M_{\mathrm{PdBI}}$, which are only lower limits.

We assume a temperature of $20 \mathrm{~K}$ in all mass derivations following Motte et al. (2007). This typical temperature was measured by $\mathrm{NH}_{3}$ measurements by Wienen (2008) using the Effelsberg telescope. The emissivity adopted (Ossenkopf \& Henning 1994) is used in most studies of low-mass starformation but also for high-mass protostellar objects (e.g. Motte et al. 2003; Hunter et al. 2006; Rathborne et al. 2007; Schuller et al. 2009) and should be adequate for dense and cold gas. Alternatively, some authors use the values by Hildebrand (1983) usually in the case of warmer and more evolved objects for which the emissivities might be different. These emissivities are 3 times smaller than those of Ossenkopf \& Henning (1994) in the millimeter range, leading to masses $\sim 3$ times higher. We thus estimate that the absolute value taken for the dust opacity is uncertain by a factor 2 . 
S. Bontemps et al.: Fragmentation in the massive dense cores of Cygnus $\mathrm{X}$
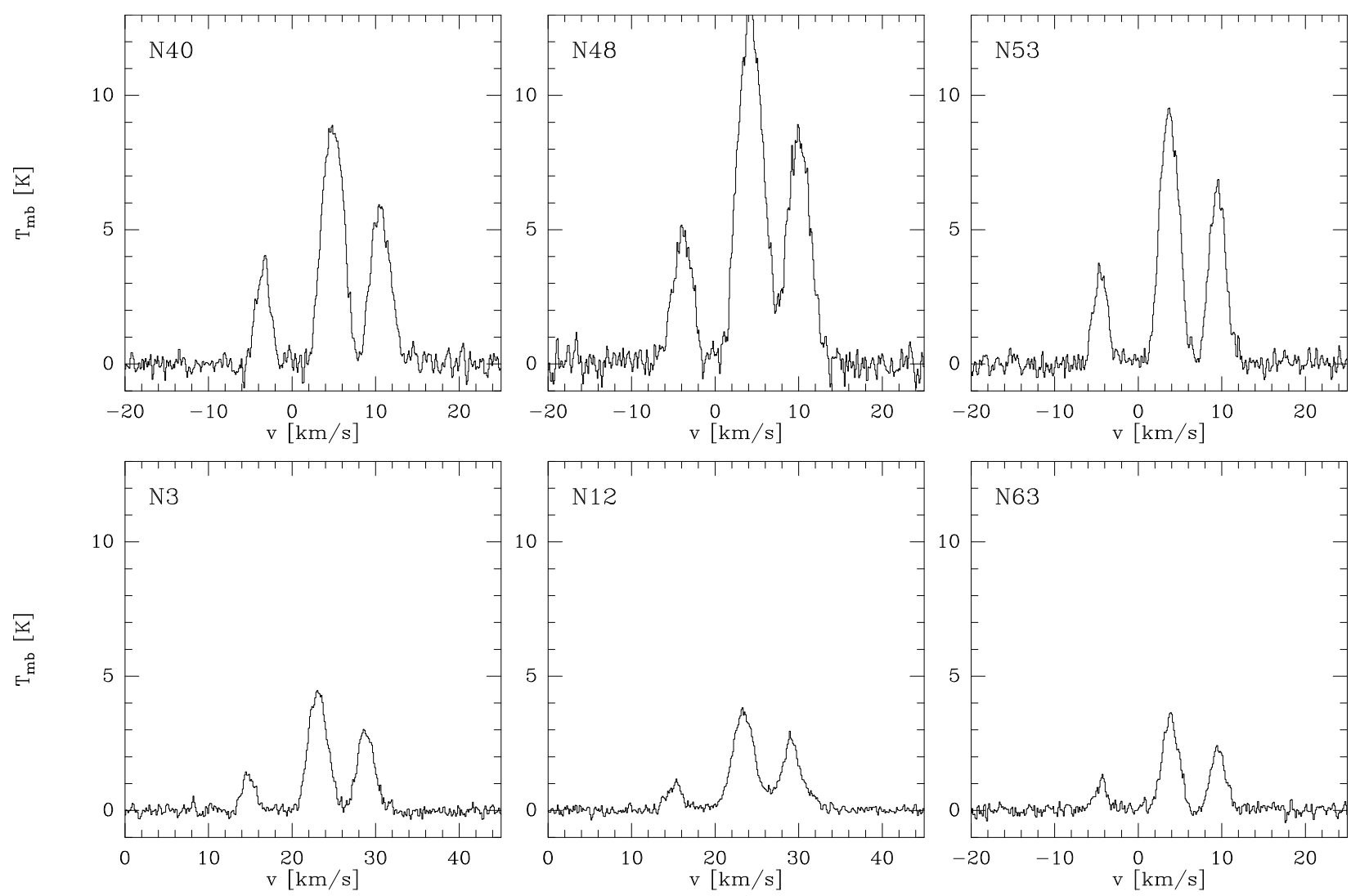

Fig. 4. $\mathrm{N}_{2} \mathrm{H}^{+}(1-0)$ spectra towards the center of each MDCs observed with the IRAM $30 \mathrm{~m}$. Note that the velocity scale is given for the weakest line (left component), which is the only isolated component of the hyperfine structure. The other 5 components of the hyperfine structure are blended and form the 2 apparently brighter groups of lines.

\section{4. $\mathrm{N}_{2} \mathrm{H}^{+} 1 \rightarrow 0$ emission towards the MDCs}

The single spectra obtained towards each MDC are shown in Fig. 4. We use these spectra to estimate the average turbulent velocity dispersion in the cores. $\mathrm{N}_{2} \mathrm{H}^{+} 1 \rightarrow 0$ is well suited line to study because it is usually optically thin and its abundance drops drastically in the lower density, warmer outer layers that tend to dominate the line widths of most other molecular tracers. Since the profiles are not always perfectly Gaussian, we did not use Gaussian fits, but directly measured the widths at half maximum of the lines. For these measurements, we only use the isolated component of the hyperfine structure (HFS), which appears as the weakest line in Fig. 4. The other 6 components are blended and form the 2 brighter groups of lines. The resulting values converted into the Gaussian 1D velocity dispersion $\sigma_{\text {turb }}$ are given in Table 3.

The fits to the 7 components of the $\mathrm{N}_{2} \mathrm{H}^{+} 1 \rightarrow 0$ multiplet can provide some indications of the opacity and of the "average" excitation temperature. The 7 relative distances and intensities of the HFS components of the $1 \rightarrow 0$ line (Caselli et al. 1995) are given as input to a simultaneous Gaussian fit to all components, assuming equal excitation temperature, using the "Method HFS" feature of GILDAS ${ }^{2}$. These fits confirm that the lines are actually optically thin with values of $\tau$ between 0.03 and 0.2 . The obtained excitation temperatures are also typically around $20 \mathrm{~K}$, which conform with $\mathrm{NH}_{3}$ observations.

\footnotetext{
${ }^{2}$ Grenoble Imaging and Line Data Analysis software.
}

\section{Discussion}

\subsection{Properties of fragmentation in low-mass, nearby protoclusters}

We review the properties of proto-stellar objects and dense cores observed in nearby low-mass star-forming regions, which are to be used as a reference to compare with the MDCs in Cygnus X to justify the definitions and assumptions applied in the remainder of the discussion.

The earliest phases of low-mass star formation correspond to the so-called pre-stellar cores and Class 0/Class I YSOs (see reviews by André et al. 2000; Ward-Thompson et al. 2007; di Francesco et al. 2007). In clustered star-forming regions such as $\rho$ Ophiuchus, Perseus, Serpens, or Orion, pre-stellar cores and envelopes of Class 0 YSOs are $\sim 0.02$ pc cloud fragments or condensations with a separation between objects of a few $1000 \mathrm{AU}$. They are usually gravitationally bound and collapsing (André et al. 2007) and have been recognized as the direct progenitors of low-mass stars in protoclusters (Motte et al. 1998). The mass distribution of these cloud fragments, which is indeed usually referred to as the core mass function (CMF), was found to have the shape of the stellar IMF but also to have the correct typical masses of the low-mass stars formed in these regions, suggesting a one-to-one correspondence between the fragments and the final stars (Motte et al. 1998; Testi \& Sargent 1998; Johnstone et al. 2000). In this view, the mass of a star would already be determined at the pre-stellar stage as a result of the fragmentation of dense cores. 
Table 3. Global properties of the MDCs derived from single dish continuum and $\mathrm{N}_{2} \mathrm{H}^{+}$line observations, and predictions for the star formation activity of a protocluster with a normal IMF and SFE $=30 \%$.

\begin{tabular}{cccccccccccc}
\hline \hline Name & $\begin{array}{c}M_{\mathrm{SD}^{a}} \\
{\left[M_{\odot}\right]}\end{array}$ & $\begin{array}{c}\text { Size }^{a} \\
{[\mathrm{AU}]}\end{array}$ & $\begin{array}{c}\left\langle n_{\mathrm{H}_{2}}\right\rangle^{a} \\
{\left[\mathrm{~cm}^{-3}\right]}\end{array}$ & $\begin{array}{c}\sigma_{\text {turb }} \\
{\left[\mathrm{km} \mathrm{s}^{-1}\right]}\end{array}$ & $\begin{array}{c}\Sigma_{\mathrm{MDC}} \\
{\left[\mathrm{g} \mathrm{cm}^{-2}\right]}\end{array}$ & $\begin{array}{c}M_{\text {vir }} \\
{\left[M_{\odot}\right]}\end{array}$ & $\begin{array}{c}\lambda_{\mathrm{J}} \\
{[\mathrm{AU}]}\end{array}$ & $\begin{array}{c}\tau_{\mathrm{ff}} \\
{[\mathrm{yr}]}\end{array}$ & $\begin{array}{c}N_{\star}{ }^{b} \\
M_{\star}^{\max c} \\
{\left[M_{\odot}\right]}\end{array}$ & $\begin{array}{c}\lambda_{\text {sep }} \\
{[\mathrm{AU}]}\end{array}$ \\
\hline CygX-N3 & 84.0 & 20300 & $5.29 \times 10^{5}$ & 0.98 & 1.45 & 54.6 & 8490 & $4.6 \times 10^{4}$ & 66 & 2.9 & 6150 \\
CygX-N12 & 86.0 & 20400 & $5.33 \times 10^{5}$ & 0.89 & 1.47 & 45.3 & 8460 & $4.6 \times 10^{4}$ & 68 & 3.0 & 3420 \\
CygX-N40 & 106.0 & 33200 & $1.52 \times 10^{5}$ & 0.82 & - & - & - & - & - & - & - \\
CygX-N48 & 197.0 & 27300 & $5.10 \times 10^{5}$ & 1.28 & 1.88 & 125 & 8640 & $4.7 \times 10^{4}$ & 156 & 5.6 & 3830 \\
CygX-N53 & 85.0 & 24000 & $3.24 \times 10^{5}$ & 0.76 & 1.05 & 38.9 & 10850 & $5.9 \times 10^{4}$ & 67 & 2.9 & 4180 \\
CygX-N63 & 58.0 & 12300 & $1.64 \times 10^{6}$ & 0.72 & 2.73 & 17.9 & 4820 & $2.6 \times 10^{4}$ & 46 & 2.2 & - \\
\hline
\end{tabular}

Notes. The de-projected average distances $\lambda_{\text {sep }}$ between the PdBI fragments is also provided. ${ }^{(a)}$ Mass, size, and average densities derived from single-dish observations by Motte et al. (2007). The average densities are however derived here in slightly different volumes than in Motte et al. (2007), inside $R_{90}$ as for the fragments (see Sect. 3.3). ${ }^{(b)}$ It includes all stars and brown dwarfs from 0.01 to $120 M_{\odot}$, among which $63 \%$ are stars (above $\left.0.08 M_{\odot}\right) .{ }^{(c)}$ Maximum stellar mass expected for a normal IMF for a global SFE of $30 \%$ on the scale of the MDC (see Sect. 4.5).

The above results may appear quantitatively different from those obtained by e.g. Alves et al. (2007), Nutter \& Ward-Thompson (2007), or Enoch et al. (2008), but these authors identified significantly larger ( $>\sim 0.1 \mathrm{pc})$ structures than the earlier works (Motte et al. 1998; Testi \& Sargent 1998). These larger structures are more likely to be dense cores in terms of size than single collapsing objects. Many of them are of low density, and all of them may not even be gravitationally bound. Their mass distribution when compared to the IMF has consistently been found to correspond to an efficiency of mass transfer into stars of $\sim 30 \%$. This star formation efficiency (hereafter SFE) may thus account mostly for the efficiency of fragmentation of a dense core into individual pre-stellar and proto-stellar condensations. In the following, we define the condensation or core formation efficiency (hereafter CFE) as the fraction of mass of a dense core found in pre- and proto-stellar envelopes (see Sect. 4.7). Interestingly, in the $\rho$ Ophiuchi protocluster, the presently observed CFE of dense cores and the total SFE measured, including the masses of the already formed stars, was also found to be of the order of $\sim 30 \%$ (Motte et al. 1998; Bontemps et al. 2001) and is identical to the value obtained from the complete investigation of nearby protoclusters with Spitzer by Evans et al. (2009).

More generally, the link between the observed CMFs and the IMF is still a highly debated issue. As already pointed out by Motte \& André (2001) and also recognized by Smith et al. (2009), the definition of true single collapsing cores is indeed partly a problem of boundaries/size of the cloud structure to be considered. The origin of the IMF probed by observed CMFs may even not allow us to differentiate between theories, especially for the high-mass part, if the shape of the observed CMFs are just a natural consequence of any formation scenario (Swift \& Williams 2008).

In analogy with the low-mass dense cores, the SFE and the CFE in the Cygnus X MDCs could therefore be of the order of $30 \%$ on the scale of $0.1 \mathrm{pc}$. In contrast, the SFE is expected to be higher and possibly close to $100 \%$ in the small-scale fragments of $0.02 \mathrm{pc}(4000 \mathrm{AU})$. We note that in the competitive accretion scenario for the formation of high-mass stars, the true single collapsing objects would not be completely decoupled from their environment because they would collect mass during their main infall/accretion phase (e.g. Smith et al. 2009, and references therein). In this case, this could even lead to an equivalent SFE higher than $100 \%$ since they would collect mass from the surrounding gas that may later be incorporated into the final star.

\subsection{Global properties of the MDCs}

The targeted MDCs are very dense cores with densities 10 times higher than in low-mass star-forming regions (see Table 4 in Motte et al. 2007). They also have total masses high enough to form a single high-mass protostar (McKee \& Tan 2003) or a whole cluster of lower mass stars (Bonnell et al. 2007).

In the first columns of Table 3, we give the properties of the MDCs directly derived from the MAMBO survey of Motte et al. (2007): the names, total masses, 1D deconvolved sizes, and average densities, along with the 1D turbulent dispersion derived from the $\mathrm{N}_{2} \mathrm{H}^{+}$line widths, and the mean surface densities $\Sigma_{\text {MDC. }}$. The values of $\Sigma_{\mathrm{MDC}}=M /\left(\pi R^{2}\right)$ are calculated for the half-mass radius for comparison with values promoted in McKee \& Tan (2003).

In the second part of Table 3, we give the quantities understood to ultimately dictate the properties of the proto-stellar fragmentation and of the subsequent star formation: the virial masses $M_{\mathrm{vir}}$, Jeans masses $M_{\mathrm{J}}$, free fall time $\tau_{\mathrm{ff}}$, the number of stars $N_{\star}$, and the maximum stellar mass $M_{\star}^{\max }$ expected to be formed in the protocluster assuming a SFE of $30 \%$ and a normal IMF (see Sect. 4.5 for details). The virial and Jeans masses, and free fall times are calculated for the average conditions in the MDCs using the classical formulation

$$
\begin{aligned}
& \lambda_{\mathrm{J}}=\sqrt{\frac{\pi}{G \rho} \frac{k T}{\mu}}=1.38 \times 10^{4} \mathrm{AU}\left(\frac{T}{10 \mathrm{~K}}\right)^{0.5}\left(\frac{n_{\mathrm{H}_{2}}}{10^{5} \mathrm{~cm}^{-3}}\right)^{-0.5} \\
& M_{\mathrm{vir}}=\frac{3 \sigma_{\mathrm{turb}}^{2} R}{G}=69.4 M_{\odot}\left(\frac{R}{0.1 \mathrm{pc}}\right)\left(\frac{\sigma_{\mathrm{turb}}}{1 \mathrm{~km} \mathrm{~s}^{-1}}\right)^{2} \\
& \tau_{\mathrm{ff}}=\sqrt{\frac{3 \pi}{32 G \rho}}=1.07 \times 10^{5} \mathrm{yr}\left(\frac{n_{\mathrm{H}_{2}}}{10^{5} \mathrm{~cm}^{-3}}\right)^{-0.5}
\end{aligned}
$$

The listed $\lambda_{\mathrm{J}}$ have been calculated for a temperature of $20 \mathrm{~K}$ (see Sect. 3.3). The above virial mass is for a density profile in $r^{-2}$ (the value would be 5/3 larger for a flat profile), and is calculated for $R=R_{90}$ and $\sigma_{\text {turb }}$, the 1D (averaged along the line-of-sight) velocity dispersion.

The average densities in the MDCs are so high that the freefall times are significantly shorter than $10^{5} \mathrm{yr}$. This is actually shorter than the statistical timescales for low-mass protostars derived in nearby star-forming regions (see also Motte et al. 2007). The duration of the proto-stellar evolution, which corresponds to the sum of the lifetimes of Class 0 and Class I YSOs, is found 
in the range $2-7 \times 10^{5} \mathrm{yr}$ (e.g. Greene et al. 1994; Kenyon \& Hartmann 1995; Evans et al. 2009). The free-fall times are also roughly equal to the crossing times of the cores, estimated by dividing the sizes by the $3 \mathrm{D}$ velocity dispersions $\left(\sqrt{3} \times \sigma_{\text {turb }}\right)$, which are typically equal to $6 \times 10^{4} \mathrm{yr}$. The MDCs are clearly self-gravitating since the ratios $M / M_{\text {vir }}$ are all close to or even larger than 1 and up to 2 for CygX-N63. The turbulent support can therefore not prevent the cores from collapsing. The unknown magnetic field could delay the collapse but cannot prevent it. If they actually collapse in a free-fall time, the global infall rates will be of the order of a few $10^{-3} M_{\odot} /$ yr which is high enough to overcome the radiation pressure barrier in the case of a monolithic collapse of the whole MDC. Their global properties (total masses, sizes, turbulent velocity dispersion, and mean surface densities) are actually consistent with the initial conditions for the turbulence-regulated monolithic collapse proposed by McKee \& Tan (2002), except perhaps for the measured velocity dispersions which, interpreted as pure micro-turbulence, tend to be in the low range of values required to support the MDCs.

\subsection{A population of dense self-gravitating fragments}

The MDCs in Cygnus X are clearly massive and self-gravitating. They are therefore expected to contain single high-mass protostellar objects, if they exist. The PdBI maps at high resolution down to physical scales of only $1700 \mathrm{AU}(0.008 \mathrm{pc})$ should identify these proto-stellar objects.

At the moderate spatial resolution of $\sim 3^{\prime \prime}(5100 \mathrm{AU})$ at $3.5 \mathrm{~mm}$ (left panels in Figs. 2 and 3), 4 out of the 5 MDCs consist of strong, centrally concentrated emission emanating from single bright, sometimes elongated (CygX-N3 and CygX-N12), cores in the inner regions of the MDCs. But, at the highest spatial resolution of $\sim 1^{\prime \prime}(1700 \mathrm{AU})$ at $1.3 \mathrm{~mm}$ (right panels in Figs. 2 and 3), the picture drastically changes. The inner cores of CygX-N3, CygX-N12, and CygX-N53 separate into a number of roughly spherical fragments. A total of 11 of these fragments ( 3 to 4 per MDC) are found to have similar sizes and separations. Only CygX-N63 remains single ${ }^{3}$ in the $1.3 \mathrm{~mm}$ map. CygX-N48 is less centrally condensed. Its central emission divides into at least two main cores at $3.5 \mathrm{~mm}$, and possibly into 4 individual fragments at $1.3 \mathrm{~mm}$ inside these two main cores. These fragments are less spherical and seem to have smaller separations than in the other MDCs. This difference could be due to an unresolved population of weak, low-mass fragments that are confused because of crowding. Alternatively, the MDC could be younger and may not yet have built up as a highly concentrated core. Finally, CygX-N63 is single and thus represents one additional fragment. Altogether, we therefore consider that a population of 16 individual fragments has been recognized in the central regions of 5 MDCs. The remaining sources listed in Table 2 are weaker sources usually in the outskirts of the MDCs, which can be considered as more uncertain detections (a few are not confirmed at $3.5 \mathrm{~mm}$ ).

Among these 16 main individual fragments, we see that 2 have $M_{\mathrm{PdBI}}$ higher than $8 M_{\odot}(\mathrm{CygX}-\mathrm{N} 53 \mathrm{MM} 1$ and CygX-N63 $\mathrm{MM} 1)$, and 5 additional ones higher than $4 M_{\odot}(\mathrm{CygX}-\mathrm{N} 12$

\footnotetext{
3 The secondary source CygX-N63 MM2 does not seem to be convincingly separated from MM1. The comparison of the 3.5 and $1.3 \mathrm{~mm}$ maps shows that the global shape of the emission is unchanged with clear extensions of MM1 towards the south-west, east and north-west directions at both resolutions and wavelengths. The extraction of MM2 as an individual fragment seems to be caused more by this east extension than to the existence of a secondary object.
}

MM1 and MM2, CygX-N48 MM1 and MM2, and CygX-N53 MM2). Owing to the filtering of the interferometer, values of $M_{\mathrm{PdBI}}$ measure the masses of more the inner regions of the fragments than their total masses (see discussion in Sect. 4.4 below). These masses inside physical sizes of only $\sim 1000 \mathrm{AU}$ correspond to exceptionally high densities, up to a few $10^{8} \mathrm{~cm}^{-3}$ (measured in $R_{90}$ ) for the most massive ones. The resulting freefall times are small, of the order of $4000 \mathrm{yr}$. The velocity dispersion in these fragments is not known. It is however reasonable to consider that it is not larger than the average dispersions $\sigma_{\text {turb }}$ measured at the scale of the MDCs. In fact, the velocity dispersion is expected to decrease from large to small scales. According to Csengeri et al. (2010), the velocity dispersion at a smaller scale of $0.03 \mathrm{pc}(6000 \mathrm{AU})$ is reduced and is of the order of $0.7 \mathrm{~km} \mathrm{~s}^{-1}$. In the extreme case there would be no further decrease of the velocity dispersion at smaller scales, with a velocity dispersion of $0.7 \mathrm{~km} \mathrm{~s}^{-1}$, the virial mass for $R_{90}=830 \mathrm{AU}$ (which corresponds to a FWHM size of $1000 \mathrm{AU}$ in Table 2) is $2.3 M_{\odot}$ which is smaller than $M_{\mathrm{PdBI}}$ for the most massive fragments. We can therefore safely conclude that these fragments are self-gravitating, and are most probably collapsing. Another indication of the probable self-gravitating nature of these fragments is the general trend of the fragments to be spherical in the continuum maps such as if gravitation is shaping them. It is more difficult to discuss the nature of the lower mass fragments but we note that some of them (see for instance CygX-N3 MM1, MM2, and MM3 in Fig. 2) are so dense, spherical and compact that they are very good candidates to be self-gravitating objects too.

The typical separations between the fragments can be evaluated from the projected distances between the fragments. The deprojected $^{4}$ average separations $\lambda_{\text {sep }}$ between the closest neighbors of the 16 main fragments are given as the last column of Table 3. We note that the separations are of the order of $5000 \mathrm{AU}$, typical for values derived in nearby, low-mass protoclusters (see Sect. 4.1).

We have thus detected a population of self-gravitating fragments whose sizes and separations are similar to low-mass prestellar cores and YSOs in nearby protoclusters (see Sect. 4.1). These fragments are most probably individual proto-stellar or pre-stellar objects more massive than those found in nearby regions. Since the selected MDCs stayed undetected in the infrared, most of these objects should still be cold and young and should thus be more pre-stellar cores or Class 0 YSOs than Class I YSOs. Also the selected MDCs are not associated with any strong radio centimeter sources from the literature. Three of them, CygX-N3, CygX-N12, and CygX-N63, were even confirmed to be devoid of $3.6 \mathrm{~cm}$ continuum sources at low levels, down to $0.1 \mathrm{mJy}$ thanks to dedicated VLA observations (Bontemps et al., in prep). No UCHII region has therefore yet developed inside these cores. Also, several collimated CO outflows have been found to be driven by most of the massive fragments in our accompanying $\mathrm{CO}(2-1) \mathrm{PdBI}$ data. These data will be presented in a forthcoming paper entirely dedicated to the nature and evolutionary status of the individual YSOs.

\subsection{Envelope masses of the proto-stellar objects}

The sizes and masses of the fragments derived from the interferometer maps are affected by the spatial filtering due to the lack of information on large scales (typically larger than $1 / 3$ of the primary beam) but also the limitation of the deconvolution

\footnotetext{
4 We use a deprojection factor equal to $1 / \sin \left(57.3^{\circ}\right)=1.19$, which corrects for the unknown random orientation of the separation axis.
} 
algorithm for limited UV coverages and noisy phases at high frequencies $(1.3 \mathrm{~mm})$, which also affects the intermediate spatial scales (sidelobes that cannot be cleaned). On top of the higher angular resolution, the stronger spatial filtering at $1.3 \mathrm{~mm}$ partly explains why the individual small scale fragments show up so clearly at $1.3 \mathrm{~mm}$ compared to $3.5 \mathrm{~mm}$. It is particularly visible for CygX-N3 and CygX-N53 where the $3.5 \mathrm{~mm}$ maps show strong, central, slightly extended emissions which do not seem to be entirely recovered at $1.3 \mathrm{~mm}$ where only the compact fragments are well detected. We therefore expect that the masses obtained at $1.3 \mathrm{~mm}$ are only valid for the smallest scales. We should thus regard the masses $M_{\mathrm{PdBI}}$ and sizes of the fragments as lower limits of proto-stellar envelopes and pre-stellar cores.

In order to estimate what could be the true total masses of the fragments, we have extrapolated $M_{\mathrm{PdBI}}$ up to realistic sizes for such proto-stellar objects, and assuming a classical density profile in $r^{-2}$ for the envelope. The most reliable sizes are provided by what has been observed in nearby protoclusters. In the $\rho$ Ophiuchi protocluster, Motte et al. (1998) derived an average size for 58 starless millimeter condensations of the order of $3000 \mathrm{AU}$ (deconvolved $F W H M$ ) for masses in the range 0.1 to $1 M_{\odot}$. In Orion $\mathrm{B}$, the more massive condensations (masses up to $\sim 3 M_{\odot}$ ) were found to be slightly larger with an average size closer to $5000 \mathrm{AU}$ (see Fig. 4 in Motte et al. 2001). By comparing $\rho$ Ophiuchi and Orion, it seems that the cores tend to be larger in size when they are more massive. In Cygnus X, and for higher mass pre-stellar and proto-stellar cores, we could therefore expect even larger sizes for the collapsing cores. This is also similar to the predictions of McKee \& Tan (2003). We however adopt here conservative sizes ranging from 3000 to $5000 \mathrm{AU}$ as in nearby protoclusters to provide a range of expected total masses $M_{\text {env }}$ given in Table 2 . We see that 9 objects have $M_{\text {env }}$ higher than $8 M_{\odot}$ (using the average mass corresponding to a size of $4000 \mathrm{AU}$ ) and are therefore likely to form ionising OB stars (earlier than B3, $M_{\star}>8 M_{\odot}$ ).

A number of high-mass star-forming regions has already been investigated at high spatial resolution with (sub)millimeter interferometers, mostly with the IRAM PdBI and the SMA. But only a few studies have been dedicated to the earliest phases of star formation and to regions of $0.1 \mathrm{pc}$ or smaller. In IRAS 05358+3543, Beuther et al. (2007) reported the detection of two cores potentially more massive than $8 M_{\odot}$ with masses of 5-10.5 and 2.7-18.5 $M_{\odot}$, respectively, inside sizes of the same order as in the present work. One of them can be further subdivided into two objects separated by only $1700 \mathrm{AU}$, and may contain an B1 star that excites a hyper-compact HII region. In the cores embedded within IRDCs observed by Rathborne et al. (2007), three cold condensations are found with masses from 9 to $21 M_{\odot}$ and sizes ranging from 5200 to $8200 \mathrm{AU}$ (0.026 and $0.041 \mathrm{pc}$ ). Rathborne et al. (2008) discovered four additional condensations in two other IRDCs with masses ranging from 9 to $29 M_{\odot}$ but in a larger average size of $12000 \mathrm{AU}(0.06 \mathrm{pc})$. Zhang et al. (2009) investigated G28.34+0.06 where 7 massive condensations were reported with masses ranging from 22 to $97 M_{\odot}$ inside physical sizes not provided in their article but which are probably significantly larger than the observation beam of $1.2^{\prime \prime}$, which corresponds to 5800 AU. Finally, we note that in the more nearby region NGC 2264, Peretto et al. (2007) could detect two condensations of $\sim 15 M_{\odot}$ inside sizes of only 1600 and 3000 AU. The massive condensations in the MDCs of Cygnus X have therefore similar masses and sizes than in the few other regions studied so far and are definitely more massive and denser than their low-mass counterparts in nearby protoclusters (see Sect. 4.1 and references therein).

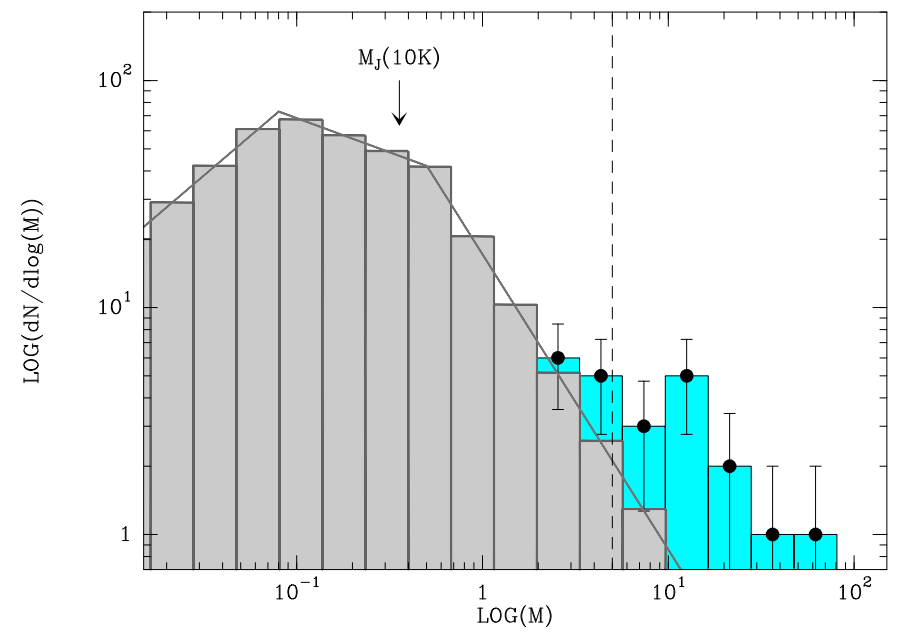

Fig. 5. Mass function of the 23 fragments in the 5 MDCs (blue histogram) using $M_{\text {env }}$ for $4000 \mathrm{AU}$ in Table 2. The error bars are for a $1 \sigma$ Poisson uncertainty. For comparison, the stellar IMF expected for a SFE of $30 \%$ on the scale of the MDCs is displayed for the Kroupa (2001) IMF for $M_{\star}$ between 0.01 and $120 M_{\odot}$ (grey histogram). The $10 \sigma$ completeness level is displayed as a vertical dashed line (see text for details).

\subsection{IMF and the CMF of the fragments}

In the simplified view that the MDCs are protoclusters, they should fragment into a population of proto-stellar objects with a mass function that mimics the IMF of stars. With a SFE of $30 \%$ typical of low-mass dense cores and a normal IMF (Kroupa 2001), a $100 M_{\odot}$ MDC would form of the order of 80 stars, with a maximum stellar mass of $\sim 3.3 M_{\odot}$. The total number of stars $N_{\star}$ and the mass of the most massive star $M_{\star}^{\max }$ expected to form in each MDC are calculated and provided in Table 3. The masses $M_{\text {env }}$ of the most massive fragments in each MDC are all systematically higher than these predictions. To aid our comparison with the expected global IMF, the mass spectrum of the 23 detected fragments in the 5 MDCs of Table 2 is plotted in Fig. 5. Our PdBI data cannot detect any low-mass fragments, and the typical $10 \sigma$ completeness $^{5}$ level is equal to $5 M_{\odot}$ for 4 of the 5 MDCs and could reach up to $\sim 10 M_{\odot}$ for CygX-N63.

Since the masses are derived consistently (at $1.3 \mathrm{~mm}$, with the same temperature and dust emissivity) for both the MDCs and the small-scale fragments, the shift of roughly a factor of three in mass between the IMF for 30\% SFE (grey histogram) and the CMF (blue histogram in Fig. 5) is significant. Thus, either the SFE is actually $30 \%$ on the scale of the fragments too or there is a significant excess of high-mass star precursors. It would be surprising if the SFE did not increase from the MDC scale down to the scale of the much denser fragments. The local SFE is expected to increase on smaller scales as a function of the average density. Moreover, the high-mass fragments dominate the local gravity and may even collect more mass during their proto-stellar phase. The effect of competitive accretion will be investigated in greater depth in a forthcoming paper on the dynamics in these MDCs (Csengeri et al. 2010).

\footnotetext{
5 The completeness level is derived at $10 \sigma$ for the typical rms of $2 \mathrm{mJy} / \mathrm{beam}$ (see Table 2), and for a size of $4000 \mathrm{AU}$. The rms is higher in CygX-N63 but it concerns only one object in the mass function of Fig. 5.
} 


\subsection{Monolithic collapse or gravo-turbulent fragmentation plus competitive accretion?}

The present observations clearly demonstrate that most of the MDCs contain fragments and should therefore be classified as protoclusters, and that they have exactly the required masses, surface densities, and line widths to be cores in monolithic collapse in the turbulence-regulated view of McKee \& Tan (2002) (see Sect. 4.2).

Only the most massive fragment, CygX-N63 MM1, seems to be a good candidate to be a turbulence regulated massive core $\left(\sigma_{\text {turb }}=0.72 \mathrm{~km} \mathrm{~s}^{-1}\right.$; see Table 3$)$. Interestingly enough, it is also the most massive single object of the sample. With a size of $0.06 \mathrm{pc}(F W H M)$ and therefore a half-mass radius of $\sim 0.03 \mathrm{pc}$, it has a size very close to the core radius predicted in McKee \& Tan (2003). Some additional investigation is required to establish the kinematic state of this core. The line width in CygX-N63 is indeed rather small for such a massive core in the quasi-static evolution view of McKee \& Tan (2002). It leads to an exceptionally large $M / M_{\text {vir }}$ ratio of 2.7 . The line width is only 2.7 times larger than the thermal width at $20 \mathrm{~K}$.

Alternatively, in the gravo-turbulent fragmentation scenario, the MDCs are expected to fragment into low-mass (Jeans mass) cores and form clusters of protostars (Dobbs et al. 2005), which may later on accumulate more mass in the central regions by competitive accretion (Bonnell \& Bate 2006). In the case of strong radiative warming from already formed massive stars, the local Jeans mass could be higher (e.g. Krumholz \& Bonnell 2009). The local temperatures are however not high enough (yet?) to have Jeans masses of the order of $10 M_{\odot}$ (see Table 3 ; Wienen 2008). We note also that turbulent fragmentation may have difficulties in forming high-mass fragments leading to CMFs with a deficit of high-mass cores (see discussions in Bonnell et al. 2007, and references therein), this issue remaining strongly debated (e.g. Padoan et al. 2007; Ballesteros-Paredes et al. 2006; Hennebelle \& Chabrier 2008). An additional process, such as micro-turbulent support or competitive accretion, might well be required to explain the formation of high-mass stars.

The observed fragmentation of the MDCs may be consistent with the gravo-turbulent fragmentation scenario. The fraction of mass in the high-mass fragments, and the level of fragmentation (number of fragments) in the MDCs are however incompatible with the pure gravo-turbulent fragmentation that would lead to a whole cluster of mostly low-mass objects. The most striking examples are CygX-N12, CygX-N53, and CygX-N63, which have 28,44 , and $100 \%$ of their total mass, respectively, in their two most massive fragments while only a few percent would be expected. According to the view of Bonnell and collaborators, a large fraction of the Cygnus X MDCs would already be in a stage after intense competitive accretion occurred to explain such high-mass objects. This might be surprising since the cores are still cold and therefore young. On the other hand, the high fraction of mass in dense fragments indicates that the selfgravity of these protoclusters is dominated by these few protostellar objects as required for an effective competitive accretion. Only a careful analysis of the dynamical state of these MDCs can help us to understand the importance of competitive accretion (see Csengeri et al. 2010).

Finally, we note that the bias of the fragmentation towards high-mass fragments could be primordial if another support such as the magnetic field were found to prevent a high degree of fragmentation as already demonstrated using MHD numerical codes for the collapse of low-mass pre-stellar cores (Hennebelle \& Teyssier 2008).

\subsection{Core formation efficiency and self-gravity}

As discussed in the previous sections, some of the MDCs tend to be sub-fragmented in only a very few massive fragments or condensations. The fractions of the single-dish emission recovered by the interferometer are exceptionally high for CygX-N12, CygX-N53, and CygX-N63 (see Sect. 3.3). This indicates that a large fraction of their mass is in small-scale structures consisting of proto-stellar cores. It also infers a high CFE for these MDCs. To quantify this effect, we evaluated the CFE as the ratio of the mass in compact, presumably self-gravitating fragments, to the total mass of the MDC. By summing the masses of all the fragments listed in Table 2, and using $M_{\mathrm{PdBI}}$ values, we derive a CFE ranging from 8 ( $\mathrm{CygX}-\mathrm{N} 3$ and $\mathrm{CygX}-\mathrm{N} 48)$ to $60 \%$ (CygX-N63). Alternatively, the CFE can be evaluated using the extrapolated masses $M_{\mathrm{env}}$ (for the average $4000 \mathrm{AU}$ size). It then ranges from 19 to more than $100 \%$ for CygX-N63. This second estimate is more indirect (extrapolated masses) but it may be closer to the true CFE as it is corrected for the spatial filtering of the interferometer. We see that, for $\mathrm{CygX}-\mathrm{N} 3$ and CygX-N48, the efficiency is below $25 \%$ and is therefore not exceptionally high. CygX-N12 has an intermediate efficiency, which might be close to $30 \%$. In contrast, CygX-N53 and CygXN63 clearly have efficiencies above $30 \%$, indicating that the mass of these 2 MDCs is dominated by proto-stellar cores. The case of CygX-N63 can be seen, in this context, as the extreme case for which all the mass is in a single centrally concentrated object.

Interestingly enough, the MDCs with the highest CFEs are those for which most of the mass is in a few massive fragments with virtually $100 \%$ of the mass in a single object for CygX-N63, 58\% of the total mass of CygX-N53 is in only two fragments $\left(36 \%\right.$ in MM1 and $22 \%$ in MM2 using $M_{\text {env }}$ for a size of 4000 AU in Table 2), and 37\% for CygX-N12 in the two most massive fragments. The gravity of these MDCs is therefore dominated by only a few gravitation wells. Moreover, we note that the most extreme MDC, CygX-N63, has the highest average density (see Table 3 ), tracing a particularly deep gravitation well. Motte et al. (1998) obtained, in $\rho$ Ophiuchus, a similar trend of an increase in CFE as a function of density (see their Fig. 7). We therefore display, in Fig. 6, the calculated CFEs as a function of the average densities on the scale of the MDCs derived in $R_{90}$ from Table 3 . For comparison, the values obtained for similar size dense cores by Motte et al. (1998) are plotted as open triangles. It is striking that the properties of Cygnus MDCs clearly confirm the trend suggested by the efficiencies in Oph A and B2 that the highest density cores have a higher fraction of their masses inside pre-stellar and proto-stellar objects. The origin of these high efficiencies should be investigated in greater detail. We can however note that the higher the average density, the shorter the free-fall time is with the possible consequence that a global collapse on the scale of the core is expected for the highest density cores. In this simplified view, the clear trend in Fig. 6 could correspond to the transition from low density cores in the standard, large-scale equilibrium between turbulent magnetic pressures and gravity, and dense cores dominated by their self-gravity leading to a virtually $100 \%$ efficiency in mass transfer to single or to a very few collapsing proto-stellar cores. 


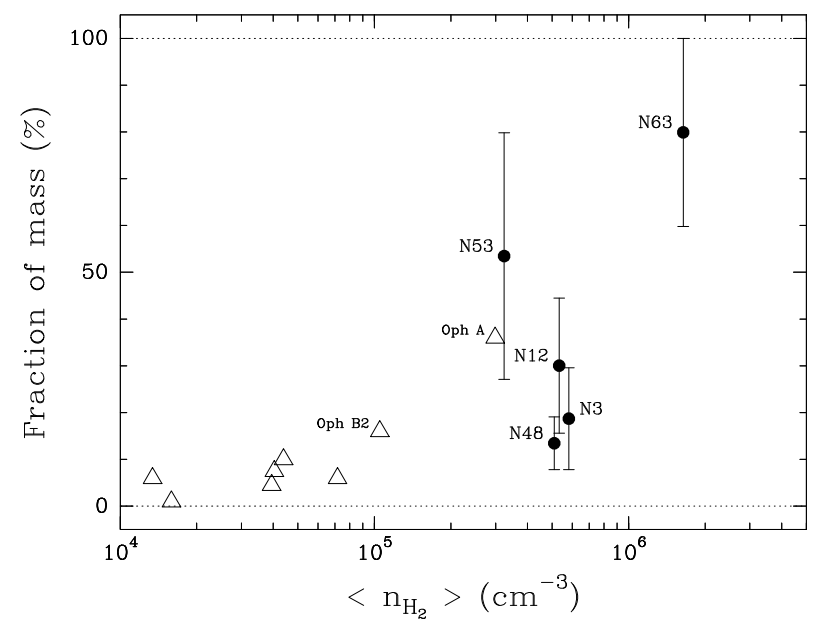

Fig. 6. Core/condensation formation efficiency (CFE) as a function of the average densities of dense cores of similar sizes ( FWHM $0.1 \mathrm{pc}$ ) but of different masses in Cygnus MDCs (filled symbols) and in $\rho$ Ophiuchus (open triangles; Motte et al. 1998). The $\rho$ Ophiuchus densities have been re-evaluated in the way we derived them in the present paper for a proper comparison. A transition from low efficiency cores to possible, single collapsing cores is observed from a few $10^{4}$ to a few $10^{6} \mathrm{~cm}^{-3}$. The displayed ranges (uncertainty bars) for the Cygnus cores correspond to the range between the sums of $M_{\mathrm{PdBI}}$ and of $M_{\mathrm{env}}$ from Table 2. To help the clarity of the plot, the displayed location of CygX-N3 corresponds to its average density shifted by a factor of 1.1 .

\subsection{Primordial mass segregation in protoclusters?}

In the young stellar clusters, such as the Orion Nebula Cluster, the highest mass members tend to be found only in their central, densest regions (Hillenbrand \& Hartmann 1998). For the youngest and not too rich clusters, the observed mass segregation cannot be explained entirely by early dynamical relaxation and is interpreted as evidence of a primordial mass segregation produced by the star/cluster formation process itself (see also Bonnell et al. 2007, and references therein). For the three most extreme MDCs discussed in this work (CygX-N12, CygX-N53, and CygX-N63), it is clear that the mass function of the protostellar objects is biased towards high masses. For these 3 cores, there is even not enough mass left to form a large population of low-mass stars to compensate for the expected excess of highmass stars (see Sect. 4.5).

If the three most extreme MDCs of our sample correspond to the mass-segregated, inner regions of clusters in the act of formation, where and when do the low-mass stars form? Since the most extreme MDCs do not have enough mass left, some surrounding gas will have to be collected in the near future from lower density gas around the MDCs to form the lower mass stars. We note however that this star-forming gas cannot be located too far from the center of the MDCs in order to ensure that the formed stars can incorporate the final cluster before it becomes visible in the infrared and establishes a normal IMF, i.e. typically before $0.5 \mathrm{Myr}$ (the age of the youngest, discovered embedded clusters, e.g. Lada \& Lada 2003). With a typical velocity dispersion of $0.9 \mathrm{~km} \mathrm{~s}^{-1}$ (see Table 3), a crossing time of $0.5 \mathrm{Myr}$ infers a distance of $0.45 \mathrm{pc}$. The observed MDCs of Cygnus X are all embedded in larger scale clumps that have total masses ranging from 260 to $7300 M_{\odot}$, sizes ranging from 0.25 to $0.74 \mathrm{pc}$ $(F W H M)$, and average densities ranging from $2.5 \times 10^{4}$ to $1.3 \times$ $10^{5} \mathrm{~cm}^{-3}$ (see Table 2 in Motte et al. 2007). Therefore, about several hundreds of solar masses of gas of relatively high density are available within a radius of $0.45 \mathrm{pc}$ around each MDC, which is a priori a large enough mass reservoir to form the missing population of low-mass stars and recover a normal IMF. Alternatively, a large, already formed population of low-mass stars and protostars might be present in the immediate surroundings of the MDCs. The spatial distribution of IR excess sources in the vicinity of the DR21 region by Kumar et al. (2007) indicates that these populations of young low-mass stars do exist in the regions surrounding CygX-N53 and CygX-N48. A more detailed and dedicated study, including the other MDCs, is however required to fully investigate this scenario, which will be possible using Spitzer data from the legacy program towards Cygnus X (Hora et al. 2009).

\section{Conclusions}

We have been imaging at high spatial resolution with the IRAM PdBI interferometer, the most massive IR-quiet dense cores discovered by Motte et al. (2007) in Cygnus X. These MDCs are among the best targets to investigate the origin, and the earliest phases of the formation of both high-mass stars and OB clusters. From this dataset, we have been able to reach the following conclusions and results:

1. All except one of the MDCs (CygX-N40) have been found to be bright and easily detected with the PdBI in a beam of $\sim 3.0^{\prime \prime} \times 2.3^{\prime \prime}$ at $3.5 \mathrm{~mm}$, and $\sim 1.1^{\prime \prime} \times 0.9^{\prime \prime}$ at $1.3 \mathrm{~mm}$.

2. There is a striking morphological difference between the lower spatial resolution maps at $3.5 \mathrm{~mm}$ and the high resolution maps at $1.3 \mathrm{~mm}$ for 3 of the 5 MDCs. While at $3.5 \mathrm{~mm}$, the MDCs are dominated by bright central emission, at $1.3 \mathrm{~mm}$, this emission is clearly separated into a population of compact fragments with typical separations of $5000 \mathrm{AU}$ and sizes larger than $1000 \mathrm{AU}$.

3. All except one of the MDCs (CygX-N63) are subfragmented. CygX-N63 consists of a single, centrally concentrated object down to the smallest scales. It may actually be a single proto-stellar object with an envelope mass as high as $\sim 60 M_{\odot}$ and may therefore form an $\mathrm{O}$ star.

4. A population of 23 compact dense fragments are recognized as probably self-gravitating, proto-stellar objects. Among them, 9 objects are good candidates to be true, individual high-mass protostars in the sense that they are precursors of OB stars.

5. The targeted MDCs of Cygnus $X$ have globally the properties proposed as initial conditions by McKee \& Tan (2003) for the turbulence-regulated, monolithic collapse. But these MDCs are actually almost all sub-fragmented. Only CygX-N63 was found to be single.

6. On the other hand, the properties of the fragmentation of the MDCs are inconsistent with a pure gravo-turbulent fragmentation. A large fraction of the mass of 3 out of 5 MDCs is located in a few, high-mass objects that would not be expected in a Jeans mass fragmentation. The local Jeans masses are too low, of the order of $1 M_{\odot}$. In a similar way, the core mass function of the fragments is globally (for the $5 \mathrm{MDCs}$ ) biased towards high-masses relative to a normal IMF-like CMF with a global SFE of $30 \%$.

7. We found a clear trend in which the CFE increases with the average density of the MDCs. Since the density is directly related to self-gravity, we interpreted this result as being due to the larger effect of self-gravity in the densest cores leading to their global collapse.

8. At the same time, as the density increases, the fragmentation tends to lead to a fewer number of predominantly high-mass 
fragments in the central part of the cores. The central regions of 3 of the 5 MDCs are dominated by a few (one or two) massive objects that may lead to regions with a clear mass segregation. This mass segregation is proposed to correspond to the primordial mass segregation of young stellar clusters.

Acknowledgements. We would like to thank the anonymous referee for its critical review of the article and for the many useful comments and suggestions which have lead to a significant improvement of the text. Part of this work was supported by the ANR (Agence Nationale pour la Recherche) project "PROBeS", number ANR-08-BLAN-0241. T. Csengeri acknowledges support from the FP6 Marie-Curie Research Training Network "Constellation: the origin of stellar masses" (MRTN-CT-2006-035890).

\section{References}

Alves, J., Lombardi, M., \& Lada, C. J. 2007, A\&A, 462, L17

André, P., Ward-Thompson, D., \& Barsony, M. 1993, ApJ, 406, 122

André, P., Ward-Thompson, D., \& Barsony, M. 2000, Protostars and Planets IV, 59

André, P., Belloche, A., Motte, F., \& Peretto, N. 2007, A\&A, 472, 519

Bacmann, A., André, P., Puget, J.-L., et al. 2000, A\&A, 361, 555

Ballesteros-Paredes, J., Gazol, A., Kim, J., et al. 2006, ApJ, 637, 384

Beuther, H., Schilke, P., Menten, K. M., et al. 2002, ApJ, 566, 945

Beuther, H., Leurini, S., Schilke, P., et al. 2007, A\&A, 466, 1065

Bonnell, I. A., \& Bate, M. R. 2006, MNRAS, 370, 488

Bonnell, I. A., Larson, R. B., \& Zinnecker, H. 2007, in Protostars and Planets V, ed. B. Reipurth, D. Jewitt, \& K. Keil, 149

Bontemps, S., André, P., Kaas, A. A., et al. 2001, A\&A, 372, 173

Caselli, P., Myers, P. C., \& Thaddeus, P. 1995, ApJ, 455, L77

Chabrier, G. 2003, PASP, 115, 763

Chandler, C. J., Gear, W. K., \& Chini, R. 1993, MNRAS, 260, 337

Csengeri, T., Bontemps, S., Schneider, N., Motte, F., \& Dib, S. 2010, A\&A, submitted

di Francesco, J., Evans, II, N. J., Caselli, P., et al. 2007, in Protostars and Planets V, ed. B. Reipurth, D. Jewitt, \& K. Keil, 17

Dobbs, C. L., Bonnell, I. A., \& Clark, P. C. 2005, MNRAS, 360, 2

Downes, D., \& Rinehart, R. 1966, ApJ, 144, 937

Enoch, M. L., Evans, II, N. J., Sargent, A. I., et al. 2008, ApJ, 684, 1240

Evans, II, N. J., Rawlings, J. M. C., Shirley, Y. L., \& Mundy, L. G. 2001, ApJ, 557, 193

Evans, N. J., Dunham, M. M., Jørgensen, J. K., et al. 2009, ApJS, 181, 321

Falgarone, E., Hily-Blant, P., \& Levrier, F. 2004, Ap\&SS, 292, 89

Faúndez, S., Bronfman, L., Garay, G., et al. 2004, A\&A, 426, 97

Foster, P. N., \& Chevalier, R. A. 1993, ApJ, 416, 303

Greene, T. P., Wilking, B. A., André, P., Young, E. T., \& Lada, C. J. 1994, ApJ, 434,614

Hennebelle, P., \& Chabrier, G. 2008, ApJ, 684, 395

Hennebelle, P., \& Teyssier, R. 2008, A\&A, 477, 25

Hennebelle, P., Pérault, M., Teyssier, D., \& Ganesh, S. 2001, A\&A, 365, 598

Henriksen, R., Andre, P., \& Bontemps, S. 1997, A\&A, 323, 549

Hildebrand, R. H. 1983, QJRAS, 24, 267

Hillenbrand, L. A., \& Hartmann, L. W. 1998, ApJ, 492, 540

Hora, J. L., Bontemps, S., Megeath, S. T., et al. 2009, in BAAS, 41, 498

Hunter, T. R., Brogan, C. L., Megeath, S. T., et al. 2006, ApJ, 649, 888
Johnstone, D., Wilson, C. D., Moriarty-Schieven, G., et al. 2000, ApJ, 545, 327

Kenyon, S. J., \& Hartmann, L. 1995, ApJS, 101, 117

Klessen, R. S. 2001, ApJ, 556, 837

Kroupa, P. 2001, MNRAS, 322, 231

Krumholz, M. R., \& Bonnell, I. A. 2009, in Structure Formation in Astrophysics, ed. G. Chabrier (Cambridge University Press), 288

Kumar, M. S. N., Davis, C. J., Grave, J. M. C., Ferreira, B., \& Froebrich, D. 2007, MNRAS, 374, 54

Lada, C. J., \& Lada, E. A. 2003, ARA\&A, 41, 57

Larson, R. B. 1969, MNRAS, 145, 271

Larson, R. B. 1981, MNRAS, 194, 809

Le Duigou, J.-M., \& Knödlseder, J. 2002, A\&A, 392, 869

McKee, C. F., \& Tan, J. C. 2002, Nature, 416, 59

McKee, C. F., \& Tan, J. C. 2003, ApJ, 585, 850

Molinari, S., Brand, J., Cesaroni, R., \& Palla, F. 1996, A\&A, 308, 573

Motte, F., \& André, P. 2001, in From Darkness to Light: Origin and Evolution of Young Stellar Clusters, ed. T. Montmerle, \& P. André, ASP Conf. Ser., 243, 301

Motte, F., Andre, P., \& Neri, R. 1998, A\&A, 336, 150

Motte, F., André, P., Ward-Thompson, D., \& Bontemps, S. 2001, A\&A, 372, L41

Motte, F., Schilke, P., \& Lis, D. C. 2003, ApJ, 582, 277

Motte, F., Bontemps, S., Schilke, P., et al. 2007, A\&A, 476, 1243

Mueller, K. E., Shirley, Y. L., Evans, II, N. J., \& Jacobson, H. R. 2002, ApJS, 143,469

Nutter, D., \& Ward-Thompson, D. 2007, MNRAS, 374, 1413

Ossenkopf, V., \& Henning, T. 1994, A\&A, 291, 943

Padoan, P., \& Nordlund, A. 2002, ApJ, 576, 870

Padoan, P., Nordlund, A., Kritsuk, A. G., Norman, M. L., \& Li, P. S. 2007, ApJ, 661, 972

Peretto, N., \& Fuller, G. A. 2009, A\&A, 505, 405

Peretto, N., Hennebelle, P., \& André, P. 2007, A\&A, 464, 983

Pillai, T., Wyrowski, F., Carey, S. J., \& Menten, K. M. 2006, A\&A, 450, 569

Plume, R., Jaffe, D. T., Evans, II, N. J., Martin-Pintado, J., \& Gomez-Gonzalez, J. 1997, ApJ, 476, 730

Rathborne, J. M., Jackson, J. M., \& Simon, R. 2006, ApJ, 641, 389

Rathborne, J. M., Simon, R., \& Jackson, J. M. 2007, ApJ, 662, 1082

Rathborne, J. M., Jackson, J. M., Zhang, Q., \& Simon, R. 2008, ApJ, 689, 1141

Schneider, N., Bontemps, S., Simon, R., et al. 2006, A\&A, 458, 855

Schneider, N., Simon, R., Bontemps, S., Comerón, F., \& Motte, F. 2007, A\&A, 474,873

Schneider, N., Csengeri, T., Bontemps, S., et al. 2010, A\&A, in press

Schuller, F., Menten, K. M., Contreras, Y., et al. 2009, A\&A, 504, 415
Simon, R., Jackson, J. M., Rathborne, J. M., \& Chambers, E. T. 2006, ApJ, 639, 227

Smith, R. J., Longmore, S., \& Bonnell, I. 2009, MNRAS, 400, 1775

Stutzki, J., \& Guesten, R. 1990, ApJ, 356, 513

Swift, J. J., \& Williams, J. P. 2008, ApJ, 679, 552

Testi, L., \& Sargent, A. I. 1998, ApJ, 508, L91

Vazquez-Semadeni, E. 1994, ApJ, 423, 681

Ward-Thompson, D., Scott, P. F., Hills, R. E., \& Andre, P. 1994, MNRAS, 268, 276

Ward-Thompson, D., André, P., Crutcher, R., et al. 2007, in Protostars and Planets V, ed. B. Reipurth, D. Jewitt, \& K. Keil, 33

Whitworth, A., \& Summers, D. 1985, MNRAS, 214, 1

Wienen, M. 2008, in Diplomarbeit, University of Bonn

Williams, J. P., Blitz, L., \& McKee, C. F. 2000, Protostars and Planets IV, 97

Yorke, H. W., \& Sonnhalter, C. 2002, ApJ, 569, 846

Zhang, Q., Wang, Y., Pillai, T., \& Rathborne, J. 2009, ApJ, 696, 268 